\title{
Comparison of propofol and ketofol for sedation in patients undergoing shoulder surgery with interscalene and suprascapular nerve blocks
}

\author{
Yasemin Akcaalan ${ }^{1}$, Mehmet Sahap ${ }^{2} \bowtie$, Handan Gulec ${ }^{2} \bowtie$, Eyup Horasanli ${ }^{2} \bowtie$, \\ Mahmut Ugurlu ${ }^{3} \bowtie$, Serhat Akcaalan ${ }^{3} \bowtie$ \\ Author affiliations: \\ 1. \{ORCID:0000-0003-0011-3489\}, Anesthesia and Reanimation Department, Ankara City Hospital, Ankara, Turkey. \\ 2. Anesthesia and Reanimation Department, Ankara Yildirim Beyazit University, Ankara, Turkey. \\ 3. Orthopedics and Traumatology Department, Ankara Yildirim Beyazit University, Ankara, Turkey. \\ Correspondence: Yasemin Akcaalan; Phone: +90 3125526000 / +90 5426197407; E-mail: ypiskinel@gmail.com
}

\section{Abstract}

Introduction and Purpose: We compared propofol and ketofol for sedation in patients who underwent shoulder arthroscopy under anesthesia with interscalene and suprascapular blocks. We evaluated both of agents the intraoperative hemodynamic effects, sedation efficiency, postoperative recovery times and the time to discharge to the service. We aimed to achieve an effective sedation with stable hemodynamics, resulting in rapid recovery with early onset.

Methodology: The study was carried out prospectively in a double-blind randomized study on ASA I and II patients aged 18-65, total of 42 shoulder surgeries planned. Anesthesia was provided to the patients by performing interscalene and suprascapular nerve block with USG. Group 1 (Propofol group), $1 \mathrm{mg} / \mathrm{kg}$ propofol iv, in Group 2 (Ketofol group), a mixture of ketamine-propofol was prepared in a 1:1 ratio, $1 \mathrm{mg} / \mathrm{kg}$ ketofol iv was administered. Processing was started in both groups when Ramsey Sedation Scale (RSS) was 3. SBP, DBP, MBP, heart rate, $\mathrm{O}_{2}$ saturation, RSS, Faces scale were recorded throughout the case. The patients with an Aldrete score of 9 were discharged and sent to the service.

Results: A total of 42 patients undergoing planned shoulder surgeries were enrolled. No statistically significant was determined between the groups in respect of demographic data (age, gender, height, weight, ASA), operating time and postoperative length of stay in hospital. More patients required esmolol in the ketofol group compared to propofol group; $15(71.4 \%)$ vs. $7(33 \%)$ patients ( $p<0.05)$. Significant higher mean values of hemodynamic findings in the ketofol group were noted; SBP at $55 \mathrm{~min}, \mathrm{DBP}$ at $60 \mathrm{~min}, \mathrm{MBP}$ at $60 \mathrm{~min}$ and MBP on discharge (p<0.05). In the absence of esmolol, the pulse measurements at $0,1,3,25$, and $30 \mathrm{~min}$ were determined to be statistically significantly higher in the ketofol group than the propofol group $(p<0.05)$. The mean values of the SpO2 measurements were significantly lower in the ketofol group ( $p<0.05)$. No statistically significant difference was determined in respect of the postoperative modified Aldrete Scores (MAS) at any of the time points $(p>0.05)$.

Conclusion: While a deeper and higher quality sedation was provided with ketofol, we achieved rapid onset and short-acting sedation with propofol. Both agents have different superior properties and can be used safely for sedation.

Key words: Brachial plexus block, ketofol, shoulder arthroscopy, propofol, sedation

Citation: Akcaalan Y, Sahap M, Gulec H, Horasanli E, Ugurlu M, Akcaalan S. Comparison of propofol and ketofol for sedation in patients undergoing shoulder surgery with interscalene and suprascapular nerve blocks. Anaesth. pain intensive care 2022;26(1):102-109. DOI: 10.35975/apic.v26i1.1775

Received: September 24, 2021, Reviewed: October 18, 2021, Accepted: Nov 09, 2021 


\section{Introduction}

Although peripheral nerve blocks have been used very commonly, these have some disadvantages such as fear of needles, pain and remembering the procedure. Intravenous (iv) conscious sedation is used to reduce patient agitation, to increase compliance with the surgical procedure, and provide analgesia, anxiolysis and amnesia. Opioids, propofol, midazolam, ketamine, and dexmedetomidine are commonly used agents for sedoanalgesia. Propofol is frequently preferred as it can be easily titrated, has an anxiolytic, amnesic, and antiemetic effect, and has rapid onset and rapid recovery. However, there are also dose-dependent unwanted effects such as hypotension and respiratory depression. Ketamine, which is a dissociative agent, has some important properties of being sympathomimetic, analgesic and amnesic agent, and provides cardiorespiratory stability. However, there are sideeffects such as hypersalivation, hallucinations, and postoperative agitation, nausea and vomiting. The formulation of these two drugs together as a single agent allows it to be used as a safer sedative agent by each suppressing the unwanted side-effects of the other. ${ }^{1}$ Esmolol is a selective adrenergic beta blocker with rapid onset and a short half-life of approximately $9 \mathrm{~min}$, which is used in the treatment of supraventricular tachycardia. ${ }^{2}$ It has minimal side-effects in the treatment of perioperative hypertension and is effective in minimizing parasympathetic autonomic responses without any known toxicity. ${ }^{3}$ Previous studies have shown the efficacy of esmolol as the first drug for controlled hypotension. ${ }^{4,5}$

We compared the effects of propofol and ketofol in shoulder surgery applied with regional anesthesia with interscalene and suprascapular nerve blocks in regard to intraoperative and postoperative hemodynamic and respiratory parameters and sedation efficacy, and the effects on postoperative recovery and time to discharge from the recovery room to the ward.

\section{Methodology}

Approval for this study was granted by the Ethics Committee of Ankara Yildirim Beyazit University. The study was conducted on patients aged 18-65 y, evaluated as ASA I and II in the Anesthesiology and Reanimation Clinic and the Orthopedics and Traumatology Operating Theatre of Ankara City Hospital.

Patients, who did not accept regional anesthesia, had BMI > $35 \mathrm{~kg} / \mathrm{m}^{2}$, suffered from COPD, heart failure, coagulopathy, epilepsy, intracranial mass, liver failure, kidney failure, or were using theophylline or had a known allergy to the drugs used, were excluded from the study.
This double-blind, randomized, prospective study included 42 orthopedic patients who were planned to undergo shoulder surgery. The patients who met the study inclusion criteria were determined through interviews with the surgical team. The patients were randomly separated into two groups (Groups 1 and 2) using the sealed envelope method. All the patients in both groups were given detailed information about the procedure to be applied and provided informed consent.

In the preoperative block room the patients were applied first with interscalene nerve block $(7.5 \mathrm{ml} 0.5 \%$ bupivacaine $+7.5 \mathrm{ml} 2 \%$ prilocaine $+5 \mathrm{ml}$ saline at a total volume of $20 \mathrm{ml}$ ) under ultrasound guidance with a linear probe, then suprascapular nerve block (5 $\mathrm{ml} 0.5 \%$ bupivacaine $+5 \mathrm{ml}$ saline at a total volume of $10 \mathrm{ml}$ ) with a 22-gauge $10 \mathrm{~cm}$ block needle using the in-plane method. In the preoperative room, the patient was examined periodically for sensation with pinprick test and for motor examination with the modified Bromage scale to determine the success of the blocks. Loss of shoulder abduction and the development of sensory block in the C5-C6 dermatomes were accepted as findings of successful motor and sensory block.

Patients who developed successful block were transferred to the operating table for the surgical operation. The patients were monitored with electrocardiography, non-invasive blood pressure, heart rate and $\mathrm{SpO}_{2}$. Oxygen support was started as 3-41t/min via an oxygen mask for each patient. Premedication of $0.03 \mathrm{mg} / \mathrm{kg}$ was administered to all patients. Each patient was gradually moved into the appropriate head upward position by the surgical team.

Group 1(Propofol): this group was applied with $1 \mathrm{mg} / \mathrm{kg}$ propofol in 30-45 sec. When the Ramsey Sedation Scale (RSS) reached 3, the procedure was started. When necessary, an additional dose of $0.5 \mathrm{mg} / \mathrm{kg}$ propofol was administered.

Group 2 (Ketofol): $10 \mathrm{ml}$ syringe was prepared with a ketamine:propofol mixture in a $1: 1$ ratio of $5 \mathrm{mg} / \mathrm{ml}$ propofol and $5 \mathrm{mg} / \mathrm{ml}$ ketamine and this was administered iv as $1 \mathrm{mg} / \mathrm{kg}$ ketofol in 30-45 seconds. When the RSS reached 3, the procedure was started. When necessary, an additional dose of $0.5 \mathrm{mg} / \mathrm{kg}$ ketofol was administered.

The patients were monitored at 5-min intervals from arrival onwards and the hemodynamic data were recorded. When mean arterial blood pressure (MBP) was $\geq 130 / 90 \mathrm{mmHg}$ initially or at any time, an esmolol infusion was started at the dose of $100-300 \mathrm{mcg} / \mathrm{kg} / \mathrm{min}$ without a loading dose. The infusion was stopped when 


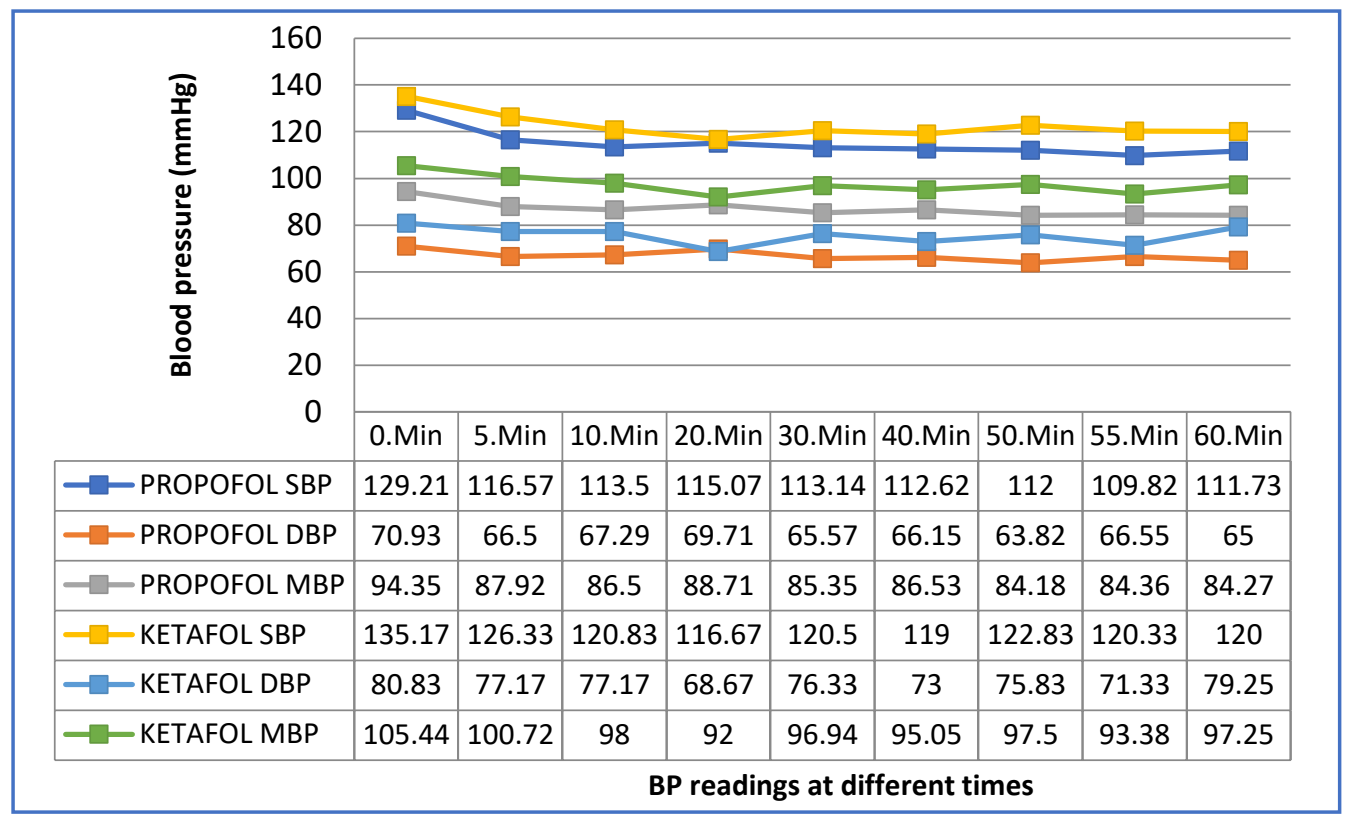

Figure 2: The hemodynamic values of patients without intraoperative esmolol use (SBP = Systolic blood pressure, MBP = Mean blood pressure, DBP = Diastolic blood pressure)

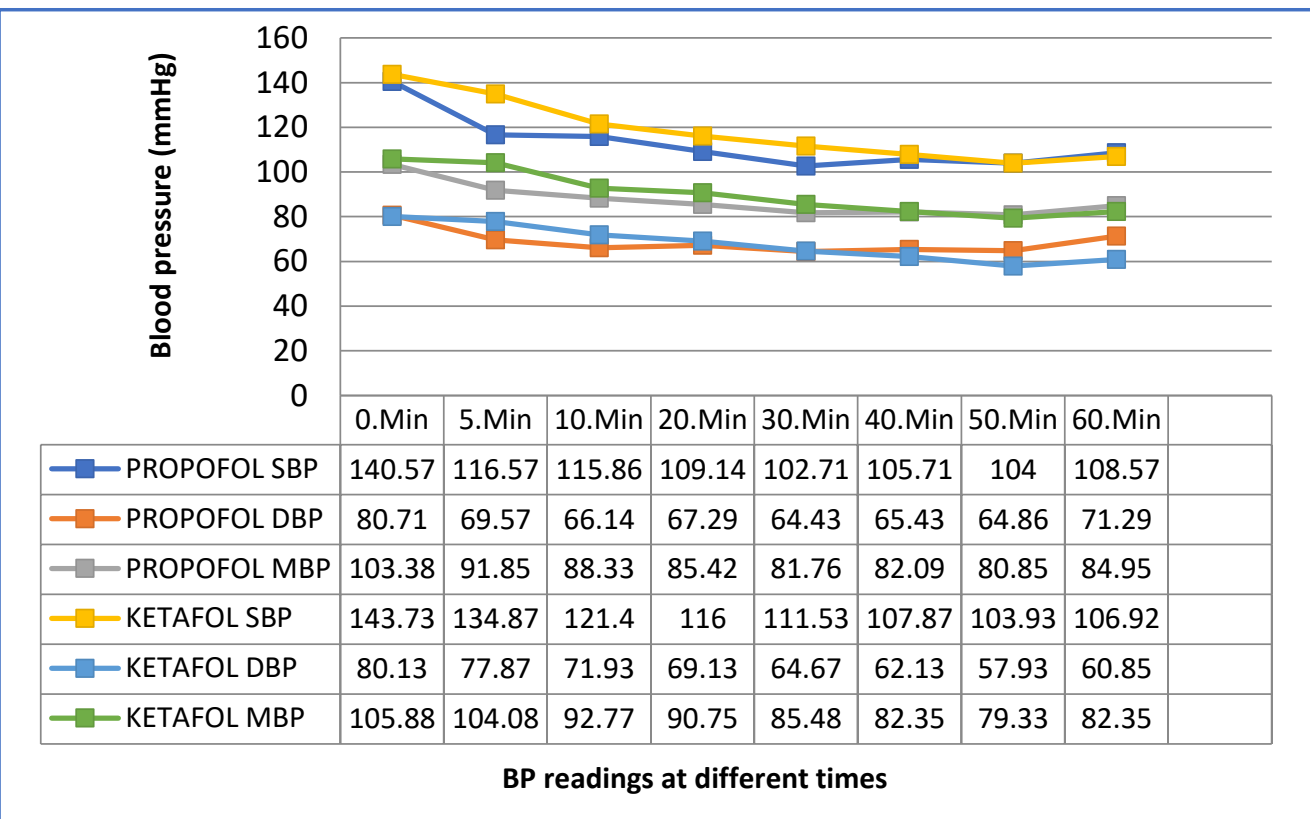

Figure 1: The hemodynamic values of patients with intraoperative esmolol use (SBP = Systolic blood pressure, MBP = Mean blood pressure, DBP = Diastolic blood pressure)

analysis

Statistical

Data obtained in the study were analyzed statistically using SPSS v. 25.0 software (Statistical Package for the Social Sciences). Conformity of the data to normal distribution was assessed with the KolmogorovSmirnov test. Descriptive statistics were stated as mean \pm standard deviation (SD), median, minimum and maximum values for continuous variables and as number (n) and percentage $(\%)$ for categorical variables. The Independent Samples t-test was applied to the comparisons of groups of continuous variables with normal distribution and the Mann Whitney U-test was used for those not showing normal distribution. For the evaluation of the difference in distribution between categorical

MBP fell to $<65 \mathrm{mmHg}$, and hemodynamic monitoring was continued in the postoperative recovery room. From arrival onwards, a record was made every $5 \mathrm{~min}$ of systolic blood pressure, diastolic blood pressure, MBP, heart rate, $\mathrm{O}_{2}$ saturation, RSS, Face Scale, modified Aldrete Score (MAS), and whether or not there were complaints of nausea and vomiting. For patients with an Aldrete score of 9 , the study was terminated and they were transferred to the ward. variables, the Chi-square test was used, and according to placement, the Chi-square test with Yates correction or the Fisher Exact test. Correlations between measurements were determined with the Pearson correlation coefficient for variables with normal distribution and with the Spearman correlation coefficient for variables not showing normal distribution. Within each group, changes over time of variables with normal distribution were evaluated with the Repeated 


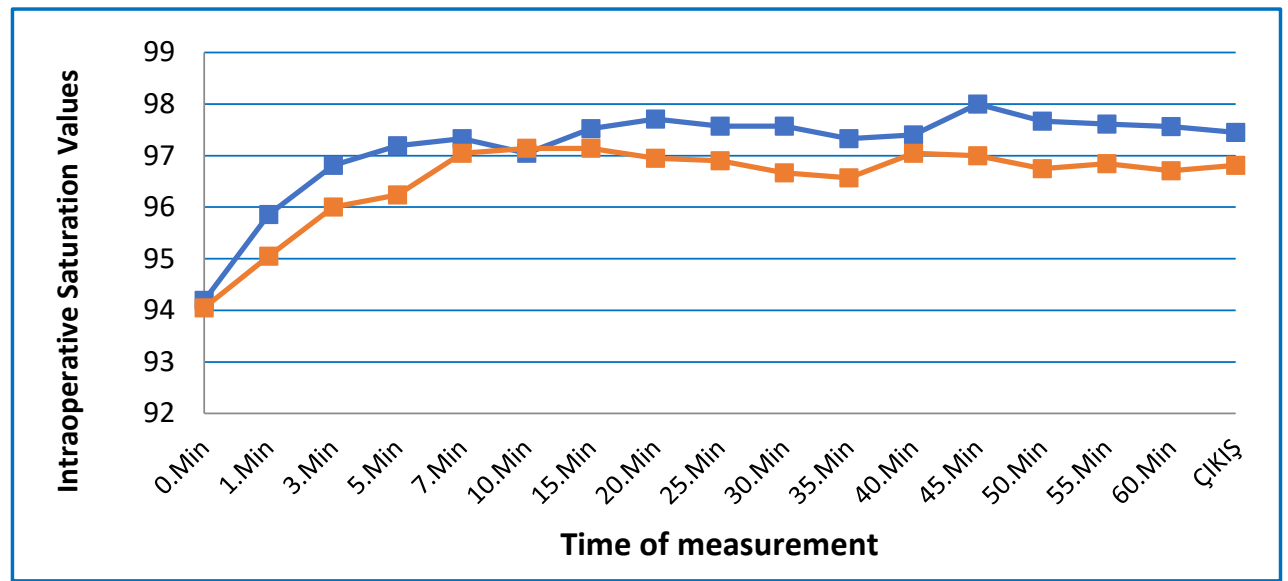

Figure 3: Comparative intraoperative SpO2 measurements

Measures ANOVA test. Variables found to be statistically significant as a change over time were applied with the post-hoc LSD test. A p $<0.05$ was accepted as statistically significant.

\section{Results}

No statistically significant difference was determined between the groups in respect of demographic data (age, gender, height, weight, ASA), operating time and postoperative length of stay in hospital. A statistically significant difference was determined between the groups in respect of the esmolol use $(\mathrm{p}<0.05)$. In the ketofol group $15(71.4 \%)$ patients required esmolol and in the propofol group, 7 (33\%) patients.

In the comparison between the groups of the measurements in the absence of esmolol, there was a statistically significant difference in the hemodynamic findings in the measurements of SBP at $55 \mathrm{~min}, \mathrm{DBP}$ at $60 \mathrm{~min}, \mathrm{MBP}$ at $60 \mathrm{~min}$ and MBP on discharge $(\mathrm{p}<0.05)$. The mean values of the ketofol group were greater at measurement points ( $\mathrm{p}>0.05)$. group. these minutes than those of the propofol group. In the presence of esmolol, statistically significant differences were determined between the groups in the measurements of SBP at $5 \mathrm{~min}, \mathrm{DBP}$ at 5 min, and MBP at 5 $\min (\mathrm{p}<0.05)$. The mean values of the ketofol group were greater at these minutes than those of the propofol group.

In the absence of esmolol, the pulse measurements at 0 , $1,3,25$, and 30 min were determined to be statistically significantly higher in the ketofol group than the propofol group ( $\mathrm{p}<0.05)$. In the presence of esmolol, there was no statistically significant difference in the heart rate values between the groups at any of the

The mean values of the $\mathrm{SpO}_{2}$ measurements at 5, 30, 50, 55 , and 60 minutes were statistically significantly lower in the ketofol group than in the propofol group ( $\mathrm{p}<0.05)$.

A statistically significant difference was determined between the groups in respect of the RSS measurements at 1,40 , and $45 \mathrm{~min}(\mathrm{p}<0.05)$. At $1 \mathrm{~min}$, the median value of the ketofol group was lower than that of the propofol group. At 40 and $45 \mathrm{~min}$, the median value of the ketofol group was higher than that of the propofol

With esmolol use, there was no statistically significant difference between the groups in respect of the postoperative

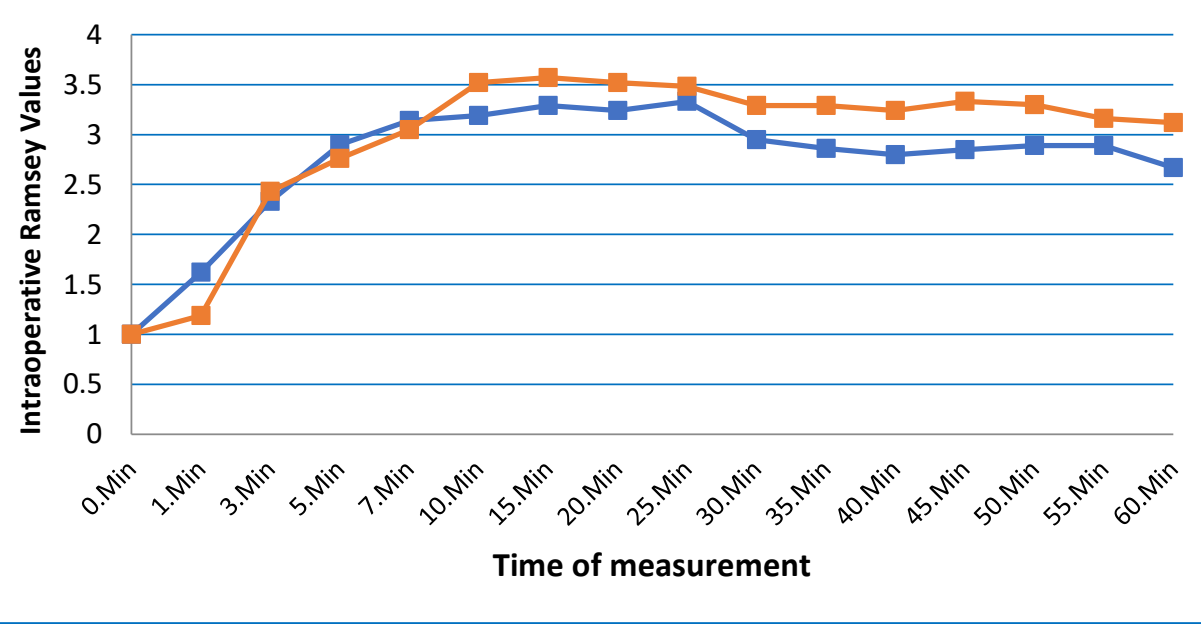

Figure 4: Comparative intraoperative Ramsay Sedation Score values measurements ( $\mathrm{p}>$ $0.05)$. In the absence of esmolol, a statistically significant difference was determined between the postoperative measurements of the groups $(\mathrm{p}<0.05)$. The mean values of SBP at $5 \mathrm{~min}$ and MBP at 5 min were significantly higher in the ketofol group than in the propofol group. No statistically significant difference

was 


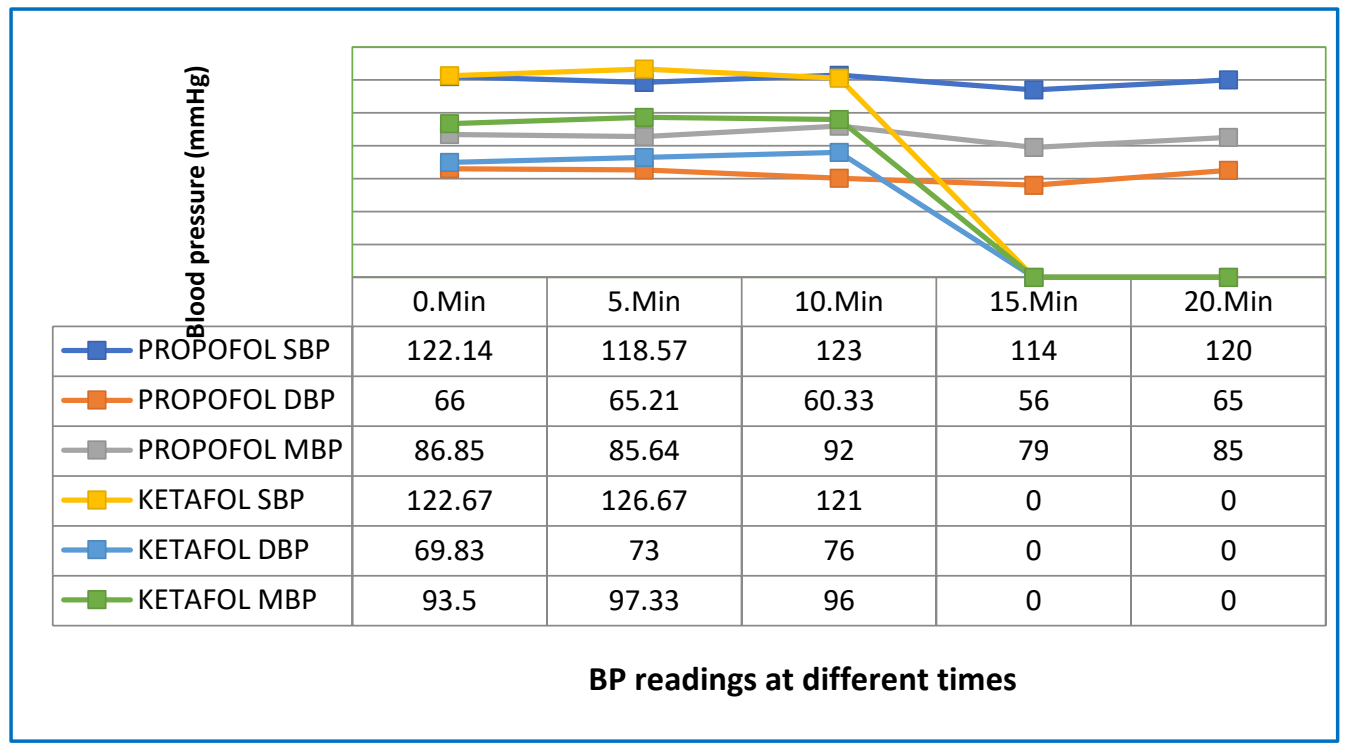

Figure 6: The postoperative hemodynamic values of patients without intraoperative esmolol use
No statistically significant difference was determined between the groups in respect of the postoperative modified Aldrete Scores (MAS) at any of the time points $(\mathrm{p}>0.05)$. A total of $25(59.5 \%)$ patients obtained MAS 9 points in the first $5 \mathrm{~min}$ postoperatively and these patients were transferred to the ward.

No statistically significant difference was determined between the groups in respect of the postoperative distribution of nausea ( $p>0.05)$. Nausea was observed in one patient of each group. No statistically significant difference was determined between the groups in respect of the distribution of the requirement for additional dose $(\mathrm{p}>0.05)$.

\section{Discussion}

When sufficient analgesia and appropriate surgical conditions are met, the performance of any surgical intervention under regional anesthesia ia accepted as a safer method compared to general anesthesia. Interscalene brachial plexus blockage for the purposes of anesthesia or analgesia has been accepted as an effective method that can be used in shoulder surgery. ${ }^{6}$

In a retrospective study by Ding $\mathrm{Y}$ et al., shoulder arthroplasties applied with general anesthesia and regional anesthesia (brachial plexus blocks) were compared. General anesthesia was preferred for obese patients and there were seen to be more respiratory complications in patients who received general anesthesia. There were fewer complications in the shoulder arthroplasties performed with isolated brachial plexus block, the need for opioid analgesia was reduced, patient satisfaction was increased, the length of stay in PACU was shorter, and early mobilization and discharge was obtained. ${ }^{7,8}$ Mirza F et al. also showed reduced consumption of anti-emetics associated with a reduced need for opioid analgesia, hemodynamic stability was maintained, no time was required for waking and extubation, excellent intraoperative muscle relaxation was provided, there was increased blood flow in the extremity with sympathetic blockage and there was a positive contribution to postoperative wound healing. ${ }^{9}$ In 


\begin{tabular}{|c|c|c|c|c|c|c|}
\hline & \multicolumn{2}{|c|}{ Postop RSS } & \multicolumn{2}{|l|}{ Groups } & \multirow[t]{2}{*}{ Total } & \multirow[t]{2}{*}{$\mathbf{P}$ valus } \\
\hline & & & Propofol & Ketofol & & \\
\hline \multirow[t]{2}{*}{$0 \mathrm{~min}$} & 1 & No. (Group \%) & $16(76.2)$ & $8(38.1)$ & $24(57.1)$ & $0.029^{*}$ \\
\hline & 2 & No. (Group \%) & $5(23.8)$ & $13(61.9)$ & $18(42.9)$ & \\
\hline \multirow[t]{2}{*}{5 mins } & 1 & No. (Group \%) & $21(100.0)$ & $13(61.9)$ & $34(81.0)$ & $0.003^{*}$ \\
\hline & 2 & No. (Group \%) & $0(0.0)$ & $8(38.1)$ & $8(19.0)$ & \\
\hline
\end{tabular}

the light of this information, interscalene and suprascapular nerve blocks were applied to the patients in the current study rather than general anesthesia.

$\mathrm{Wu} C L$ et al. reported that sedation during regional anesthesia increased patient satisfaction, ${ }^{10}$ and stated that this could be considered a means to increase patient acceptance of regional anesthesia techniques. In the current study, it was seen that the application of sedation to the patients in the beach-chair position was positively reflected in patient satisfaction in both groups. No statistically significant difference was determined between the groups in the pain evaluated with the face scale intraoperatively and postoperatively. At all the time points, the face scale was found to be 0 (no pain) in both groups.

In a study by Mittal $\mathrm{N}$ et al., the mean propofol dose and requirement for additional drug bolus was lower in the ketofol group than in the propofol group, but the difference was not found to be statistically significant. ${ }^{11}$ Andolfatto $G$ et al. found the frequency of additional dose to be significantly higher in the propofol group. ${ }^{12}$ In the current study, no significant difference was determined between the groups in respect of additional dose frequency. However, in the Andolfatto study, the procedure was started when there was RSS 5 and above in painful procedures in the Emergency Department, whereas in the current study, analgesia was provided with peripheral blockage and the procedure was started at RSS 3 and above. This can be considered to be the reason for no significant difference in the current study in respect of the requirement for additional drug dose.

In the study by Mittal et al., all the vital signs were similar at $0 \mathrm{~min}$, there was an increase in the heart rate value at 5 min compared to baseline in the ketofol group and in the propofol group, it remained stable. ${ }^{11}$ At 15, 20, 25 , and $30 \mathrm{~min}$, the heart rate value was higher in the ketofol group and the other vital signs were found to be similar in both groups at all the time points. Ferguson I et al. reported that statistically significant hypotension was seen in the propofol group. ${ }^{13}$ In the current study, there was a statistically significant difference in the intraoperative SBP and DBP measurements between the groups in the patients who did not use esmolol $(p<0.05)$. As expected, these findings originated from the ketamine sympathomimetic effect. In the patients who used esmolol, there was a statistically significant difference between the groups in respect of the preoperative SBP at $5 \mathrm{~min}, \mathrm{DBP}$ at $5 \mathrm{~min}$, and MBP at $5 \mathrm{~min}$ measurements $(\mathrm{p}<0.05)$. The mean values at these times were higher in the ketofol group than in the propofol group. In the heart rate measurements, there was no statistically significant difference in the presence of esmolol. As there was elevation only at $5 \mathrm{~min}$ in the presence of esmolol, this can be attributed to the first dose of ketamine, then the effect was suppressed with the esmolol infusion. It can be seen from the tables, that at all the other time points, although not statistically significant, the values of the ketofol group were higher than those of the propofol group.

In a study conducted with the aim of procedural sedation in the Emergency Department, Yan et al. showed that compared with propofol alone, the use of ketamine and propofol combination decreased the frequency of adverse respiratory events in patients applied with procedural sedation and analgesia. Moreover, sideeffects such as short oxygen desaturation were not found to be clinically significant. ${ }^{14}$ Ferguson I et al. reported that adverse airway effects were similar in the propofol and 1:1 ketofol groups, but there was seen to be a greater need for ventilation support with Ambu mask in the propofol group. ${ }^{13}$ In the current study, there was found to be a statistically significant difference between the groups in the saturation measurements at 5, 30, 50, 55, and $60 \min (\mathrm{p}<0.05)$.

The mean $\mathrm{SpO}_{2}$ value of the ketofol group was found to be lower than that of the propofol group. Although not statistically significant at these minutes, the RSS was found to be higher due to the deeper and higher quality sedoanalgesic effect of the ketofol agent, as can be seen in the tables. This can be considered to explain the difference in saturation between the groups at these minutes, despite the more stable effect of ketamine on the respiratory system. Saturation was seen to be around $96 \%$ in the ketofol group at the specified minutes, and even though this was not statistically significant it was evaluated as subclinical desaturation. No respiratory depression or apnea developed in any patient of either group. Strayer RJ et al. showed that compared to 
propofol, the use of ketamine was limited because of the effect of delayed recovery. ${ }^{15}$ In the study by Ferguson I et al., the Wisconsin sedation score at 6 and 9 minutes was found to be lower in ketofol, although not statistically significant, in other words, deeper sedation was provided, and this was attributed to the analgesic property of ketamine and long duration of the effect. ${ }^{3}$ The results of the current study were compatible with those of previous clinical studies.

Ferguson I et al. used the Discharge Key Score (DKS) for discharge of the patients and found that the time to reach DKS $\geq 7$ was longer in the ketofol group. ${ }^{13}$ In a review by Yan JW which included 11 randomized, controlled studies, no difference was found between the ketamine and propofol combination and propofol alone in respect of the time to discharge. ${ }^{14}$ In the current study, no statistically significant difference was determined between the groups in respect of the postoperative MAS at any of the time points $(\mathrm{p}>0.05)$. As seen in the current study and in previous clinical studies, propofol has a rapid onset of effect, whereas ketofol has a later onset but a longer-lasting effect, and deeper sedation is provided.

\section{Conclusion}

In this study, shoulder surgery was performed by applying regional anesthesia successfully with interscalene and suprascapular nerve blocks. No respiratory side-effects such as desaturation, apnea, or phrenic nerve paralysis were encountered in any of the 42 patients.

In the comparison of ketofol and propofol applied for sedation purposes with interscalene and suprascapular blocks, which are often used in shoulder surgery, despite the rapid onset of effect and rapid recovery with propofol, ketofol was found to be more effective in respect of complications, and depth and quality of intraoperative sedation. In postoperative recovery, propofol was more advantageous in respect of RSS because of the early termination of sedation, and no significant difference was found between the groups in respect of the Aldrete scores. Therefore, as the two groups both had different advantages in respect of sedation, both agents can be considered safe for use.

\section{Conflict of Interest}

No conflict of interest was declared by the authors. The authors declared that this study received no financial support.

\section{Authors' contribution}

YA: Corresponding author, manuscript writing MS, EH, MU: Manuscript editing

$\mathrm{HG}$ : Concept
SA: Concept, data collection

All authors have read the final manuscript and approve it for publication.

\section{References}

1. Miner JR, Moore JC, Austad EJ, Plummer D, Hubbard L, Gray RO. Randomized, double-blinded, clinical trial of propofol, 1:1 propofol/ketamine, and $4: 1$ propofol/ketamine for deep procedural sedation in the emergency department. Ann Emerg Med. 2015 May;65(5):479-488.e2. [PubMed] DOI: 10.1016/j.annemergmed.2014.08.046

2. Gorczynski RJ. Basic pharmacology of esmolol. Am J Cardiol. 1986;56:3F-I3F. [PubMed] DOI: 10.1016/0002-9149(85)909105

3. Menkhaus PG, Reves JG, Kissin I, Alvis JM, Govier AV, Samuelson PN, et al. Cardiovasculareffects of esmolol in anesthetized humans. Anesth Analg. 1985;64:327-34. [PubMed]

4. Blair JL, McGuire R, Killian J, Amundson G. Esmolol as primary agent for controlled hypotension (abstract). Anesth Analg 1989;68:S31.

5. Edmondson R, Del Valle O, Shah N, Wong G, Dwyer D, Matarazzo D, et al. Esmolol for potentiation of nitroprussideinducedhypotension. Impact on thecardiovascular, adrenergic, and renin-angiotensinsystems in man. Anesth Analg. 1989;69:202-6. [PubMed]

6. Özyalçin SN, Erdine S. Upper extremity somatic blocks. In: Regional Anesthesia . Nobel Medicine Bookstore: Istanbul; 2005.

7. Ding DY, Mahure SA, Mollon B, Shamah SD, Zuckerman JD, Kwon YW. Comparison of general versus isolated regional anesthesia in total shoulder arthroplasty: A retrospective propensity-matched cohort analysis. J Orthop. $2017 \mathrm{Jul}$ 21;14(4):417-424. [PubMed] DOI: 10.1016/j.jor.2017.07.002

8. Wu CL, Rouse LM, Chen JM, Miller RJ. Comparison of postoperative pain in patients receiving interscalene block or general anesthesia for shoulder surgery. Orthopedics. 2002;25:45-48. [PubMed]

9. Mirza F, Brown AR. Ultrasound-guided regional anesthesia for procedures of the upper extremity. Anesthesiol Res Pract. 2011;2011:579824. [PubMed] DOI: 10.1155/2011/579824

10. Wu CL, Naqibuddin M, Fleisher LA. Measurement of patient satisfaction as an outcome of regional anesthesia and analgesia: a systematic review. Reg Anesth Pain Med. 2001;26:196-208. [PubMed] DOI: 10.1053/rapm.2001.22257

11. Mittal N, Goyal A, Gauba K, Kapur A, Jain K. A double blind randomized trial of ketofol versus propofol for endodontic treatment of anxious pediatric patients. J Clin Pediatr Dent. 2013:37:415-20. [PubMed] 10.17796/jcpd.37.4.thv8372410714342

12. Andolfatto G, Abu-Laban RB, Zed PJ, Staniforth SM, Stackhouse S, Moadebi S, et al. Ketamine-propofol combination (ketofol) versus propofol alone for emergency department procedural sedation and analgesia: a randomized double-blind 
trial. Ann Emerg Med. 2012 Jun;59(6):504-12.e1-2. [PubMed] DOI: 10.1016/j.annemergmed.2012.01.017

13. Ferguson I, Bell A, Treston G, New L, Ding M, Holdgate A. Propofol or Ketofol for Procedural Sedation and Analgesia in Emergency Medicine-The POKER Study: A Randomized Double-Blind Clinical Trial. Ann Emerg Med. 2016 Nov;68(5):574-582.e1.

[PubMed] DOI:

10.1016/j.annemergmed.2016.05.024
14. Yan JW, McLeod SL, lansavitchene A. Ketamine-propofol versus propofol alone for procedural sedation in the emergency department: a systematic review and meta-analysis. Acad Emerg Med. 2015. [PubMed] DOI: 10.1111/acem.12737

15. Strayer RJ, Nelson LS. Adverse events associated with ketamine for procedural sedation in adults. Am J Emerg Med. 2008;26:985-1028. [PubMed] DOI: 10.1016/j.ajem.2007.12.005 